\title{
INFLUÊNCIA DE PROCESSOS ANTAGÔNICOS NO DESENVOLVIMENTO DE SISTEMA DE ANÁLISES EM FLUXO PARA A DETERMINAÇÃO ESPECTROFOTOMÉTRICA DE PROPILTIOURACIL EM MEDICAMENTOS
}

\author{
Rodolfo de Melo Magalhães Santana e Mauro Korn* \\ Departamento de Ciências Exatas e da Terra, Universidade do Estado da Bahia, R. Silveira Martins, 2555, 41195-001 Salvador \\ - BA, Brasil \\ Josué Carinhanha Caldas Santos \\ Instituto de Química e Biotecnologia, Universidade Federal de Alagoas, Campus A.C. Simões, 57072-970 Maceió - AL, Brasil
}

Recebido em 25/4/12; aceito em 13/8/12; publicado na web em 29/11/12

\begin{abstract}
INFLUENCE OF ANTAGONIC PROCESSES ON THE DEVELOPMENT OF FLOW ANALYSIS SYSTEM FOR SPECTROPHOTOMETRIC DETERMINATION OF PROPYLTHIOURACIL IN MEDICATIONS. A multi-commuted flow system was developed to determine propylthiouracil (PTU) based on the reaction of its thiol form with iminoquinone radical generated by the oxidation of $N, N$-dimethyl-p-phenylenediamine in an alkaline medium. PTU can be found in tautomeric forms and the tautomeric equilibrium was displaced to enhance the thiol form. However, the reaction product is unstable and its residence time in the flow path was carefully investigated. The proposed procedure showed good precision $(<1.5 \%)$ and a limit of detection $(3 \sigma)$ of $0.11 \mathrm{mg} \mathrm{L}^{-1}$. High recoveries were obtained in the validation test. The procedure was employed for propylthiouracil determination in medications.
\end{abstract}

Keywords: tautomeric equilibrium; antagonist processes; propylthiouracil.

\section{INTRODUÇÃO}

As novas tecnologias, os investimentos nas pesquisas, a ampliação da produção e a facilitação das condições para a aquisição de medicamentos pela população foram algumas das causas que explicam a expansão da indústria farmacêutica. Esse crescimento acarreta também a necessidade de se estabelecer protocolos para a quantificação dos fármacos presentes nos medicamentos que estão disponíveis no mercado. ${ }^{1}$ Por vezes, as farmacopeias fornecem apenas os protocolos para a determinação da pureza do princípio ativo na matéria-prima, sem trazer detalhes referentes a procedimentos que permitam a avaliação dos mesmos fármacos nas formas em que os medicamentos são comercializados. Essa dificuldade pode ser exemplificada pelo propiltiouracil (PTU), para o qual há poucos métodos analíticos disponíveis. ${ }^{2-7} \mathrm{O}$ PTU é o princípio ativo de medicamentos usados no tratamento do hipertireoidismo. Ele tem sido usado desde a década de 1950 e seu mecanismo de ação se baseia na inibição da síntese da tiroxina (T4) e da tri-iodotironina (T3), pela remoção do iodo livre numa das etapas da bioprodução desses hormônios. ${ }^{8}$ Mais recentemente, o PTU foi adotado como referência de amargor e aplicado em estudos sobre a transmissão genética da sensibilidade gustativa e em investigações sobre hábitos alimentares. ${ }^{9}$

A carência de métodos para a determinação de PTU se justifica pelo fato desse composto existir em equilíbrio tautomérico. Diferentemente de outras isomerias, a tautomeria se caracteriza por equilíbrios dinâmicos regidos pela interconversão de espécies através de rearranjos intramoleculares de átomos de hidrogênio (como no caso do PTU) ou de heteroátomos. ${ }^{10}$ As proporções entre os tautômeros numa solução são fortemente afetadas por fatores estruturais, pelo sistema solvente e pelo pH do meio. Assim, a reatividade de um tautômero será drasticamente afetada com pequenas alterações nas condições do meio reacional. ${ }^{11}$ No caso do propiltiouracil, observa-se no equilíbrio tautomérico (Figura 1) a alteração da função tioamida para um grupo tiol (I a III), aumentando a reatividade da molécula e possibilitando que reações características para compostos tiólicos venham a ocorrer.

*e-mail: mkorn@uneb.br

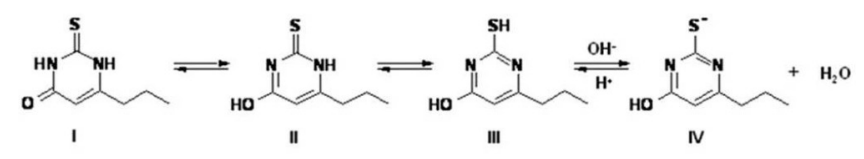

Figura 1. Estruturas canônicas relativas ao equilíbrio tautomérico do propiltiouracil (PTU)

Caso o objetivo analítico seja quantificar uma das espécies em equilíbrio tautomérico, os procedimentos analíticos serão complexos e tenderão para a aplicação de técnicas hifenadas de análise. . $^{12-15}$ Conhecer a concentração de um tautômero não necessariamente satisfaz às necessidades dos ensaios de controle de qualidade, visto que na legislação não há informação sobre as concentrações limites para cada tautômero, nem sobre as proporções entre eles.

Formas tautoméricas do ácido ibotênico (tautomerismo ceto-enólico) foram anteriormente separadas por cromatografia líquida de alta eficiência em fase reversa, mas o ácido ibotênico foi determinado empregando sistema de análises por injeção em fluxo com detecção por espectrometria de massas. ${ }^{16}$ Esse foi o único procedimento descrito na literatura que explorava as análises em fluxo para a identificação/ determinação de espécies que apresentam tautomerismo. As análises em fluxo são especialmente interessantes para aplicação em processos que dependem do tempo, como nos métodos cinéticos de análise, ${ }^{17}$ métodos quimiluminescentes; ${ }^{18} \mathrm{ou}$, generalizando, nos processos em que a espécie sob monitoração não é estável. ${ }^{19}$ Os processos de análises em fluxo têm em comum a dispersão controlada. Nos procedimentos de análises em fluxo em linha única, a mesma dispersão que favorece a formação do produto de uma reação em linha também é responsável pela diluição do produto. ${ }^{20} \mathrm{O}$ perfil de dispersão ou diluição da zona de amostra ao longo do percurso analítico pode ser alterado, devido a variações na geometria e/ou nos parâmetros hidrodinâmicos do sistema de análise. Em outras palavras, a otimização dos parâmetros de qualquer procedimento de análises em fluxo visa equacionar a influência de processos antagônicos (reação e diluição) de modo a garantir as melhores figuras de mérito possíveis.

Sistemas de análises em fluxo operam em condição de não equilíbrio, não sendo necessário aguardar longos intervalos de tempo 
para que uma reação lenta se complete. Em contrapartida, caso uma reação produza uma espécie intermediária com tempo de vida breve, a medida dessa espécie deverá ser realizada tão mais rapidamente quanto mais rápida a sua decomposição. Um evento que ocorra em intervalo de tempo curto exigirá controle mais acurado de tempo, de modo a garantir a exatidão e precisão do processo. Assim, entende-se que a exatidão e precisão dos procedimentos multicomutados de análises em fluxo serão fortemente dependentes do rígido controle de tempo de cada operação que compõe o processo. ${ }^{21}$

No presente trabalho foi desenvolvido um sistema multicomutado de análises em fluxo para a determinação espectrofotométrica de PTU em amostras de medicamentos. A configuração e os parâmetros desse sistema foram ajustados para compensar os efeitos antagônicos do deslocamento do equilíbrio tautomérico do analito para sua forma tiólica, seguida da formação de um derivado cromogênico (etapa lenta) e a conversão desse derivado cromogênico e instável do analito para o produto final (etapa rápida).

\section{PARTE EXPERIMENTAL}

\section{Equipamentos}

O sistema multicomutado de análises em fluxo foi composto por uma bomba peristáltica Minipuls 3 (Gilson, Villers le Bel, França); espectrofotômetro Femto 700 Plus (São Paulo, Brasil) equipado com célula de fluxo em borossilicato com $10 \mathrm{~mm}$ de caminho óptico e volume interno de $80 \mu \mathrm{L}$ da Hellma (Alemanha); quatro válvulas solenoides de três vias da NResearch modelo 161T031 (West Caldwell, EUA); linhas de fluxo e bobina de mistura construída com tubo de polietileno com diâmetro interno de $0,8 \mathrm{~mm}$; microcomputador equipado com interface eletrônica PCL-711S (Advantech, Taiwan, China). Um software para o controle das válvulas solenoide e para a aquisição de sinais gerados pelo espectrofotômetro foi desenvolvido em QuickBasic 4.5 (Microsoft).

\section{Reagentes, soluções e amostras}

Os reagentes usados tinham grau analítico de pureza e suas soluções foram sempre preparadas com água desionizada. Água foi usada como fluido transportador em todos os ensaios realizados no sistema multicomutado de análises em fluxo.

Solução estoque de propiltiouracil $100 \mathrm{mg} \mathrm{L}^{-1}$ foi preparada pela dissolução de $10 \mathrm{mg}$ de PTU (Galena, São Paulo) em $5 \mathrm{~mL}$ de metanol (F. Maia, Brasil), seguida da adição de água até atingir o volume de $100 \mathrm{~mL}$. Soluções de referência de propiltiouracil na faixa de concentração entre 2,5 e $30 \mathrm{mg} \mathrm{L}^{-1}$ foram preparadas pela diluição apropriada da solução estoque com água.

Para a determinação de PTU foram utilizadas soluções aquosas de $N, N$-dimetil-p-fenilenodiamina (DMPD, Aldrich, Alemanha) e $\mathrm{K}_{3} \mathrm{Fe}(\mathrm{CN})_{6}$ (Quimex, Brasil). Solução aquosa de DMPD 0,5 mmol $\mathrm{L}^{-1}$ foi diariamente preparada pela dissolução de $11,60 \mathrm{mg}$ de sulfato de DMPD em $100 \mathrm{~mL}$ de água. Após o ajuste do volume e homogeneização, a solução de DMPD foi transferida para frasco âmbar para evitar sua fotodecomposição. Uma solução $0,15 \%(\mathrm{~m} / \mathrm{v}) \mathrm{K}_{3} \mathrm{Fe}(\mathrm{CN})_{6}$ foi preparada pela dissolução de $75 \mathrm{mg}$ de $\mathrm{K}_{3} \mathrm{Fe}(\mathrm{CN})_{6}$ em $50 \mathrm{~mL}$ de solução tampão borato $0,2 \mathrm{~mol} \mathrm{~L}^{-1}$ a pH 10 .

As amostras de medicamentos produzidas por diferentes laboratórios farmacêuticos foram adquiridas no comércio local. As amostras analisadas continham em suas composições $100 \mathrm{mg}$ de PTU, mas seus excipientes eram variados (amido de milho, carbonato de cálcio, estearato de magnésio, povidona, entre outros). Para a determinação da massa média de cada comprimido foram medidas individualmente as massas de 10 comprimidos aleatoriamente escolhidos de um dado lote de cada medicamento. ${ }^{22}$ Em seguida, os 10 comprimidos de um mesmo medicamento foram triturados. $\mathrm{O}$ material finamente dividido foi quarteado e, então, foram tomadas alíquotas de cada amostra de forma que a massa de PTU nas alíquotas correspondesse a ca. $10 \mathrm{mg}$. À alíquota de cada medicamento foram adicionados 5 $\mathrm{mL}$ de metanol e, após vigorosa mistura, a dispersão foi submetida à filtração. Finalmente, o filtrado foi recolhido em balão volumétrico com capacidade para $100 \mathrm{~mL}$ e o volume completado com água até o menisco. Assim, as soluções de PTU obtidas a partir das alíquotas dos diferentes medicamentos apresentavam concentração final de ca. $100 \mathrm{mg} \mathrm{L}^{-1}$.

Testes de interferência foram realizados com tioureia, até concentração de $150 \mathrm{mg} \mathrm{L}^{-1}$. Foi preparada solução estoque para os testes de interferência do método pela dissolução de $20 \mathrm{mg}$ de tioureia (Merck, Alemanha) em 100 mL de água.

\section{Procedimentos}

O diagrama de fluxo proposto é apresentado na Figura 2. Para a limpeza das linhas, todas as válvulas foram desativadas para que somente o transportador (água) fluísse pelo percurso analítico a vazão de 2,8 $\mathrm{mL} \mathrm{min}^{-1}$. Para a inserção de uma alíquota de amostra (ou de uma das soluções de referência) no percurso analítico, as válvulas $\mathrm{V}_{1}$ e $\mathrm{V}_{2}$ foram simultaneamente ativadas de forma a interromper a inserção do carregador para que apenas a solução da amostra fluísse

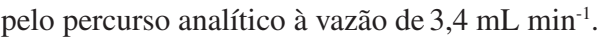
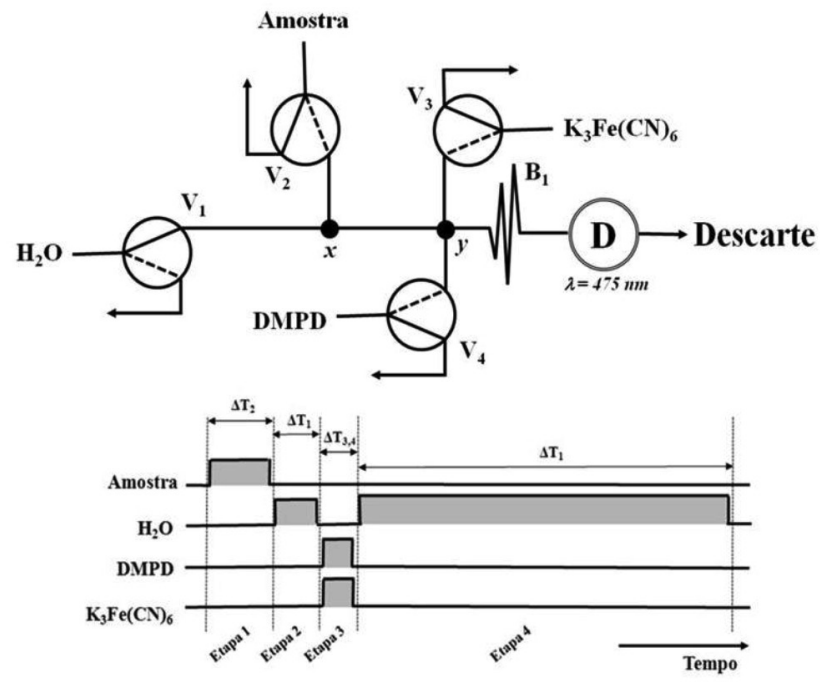

Figura 2. Representação do sistema multicomutado de análises em fluxo desenvolvido para a determinação espectrofotométrica de PTU em medicamentos. $V_{1}, V_{2}, V_{3}$ e $V_{4}=$ válvulas solenoides de 3 vias; $y$ e $x=$ confluências; $B 1=$ bobinas de mistura $(60 \mathrm{~cm}), D=$ detector espectrofotométrico $(\lambda=475$ $n m) ; \Delta T_{1}, \Delta T_{2}, \Delta T_{3}$ e $\Delta T_{4}=$ intervalos de tempo de ativação/desativação das válvulas solenoides

Nos ensaios preliminares foi observado aumento significativo do sinal analítico pela inserção concomitante das alíquotas dos reagentes (DMPD e ferricianeto) após a alíquota da amostra ser inserida no percurso analítico. Assim, para a inserção simultânea das soluções dos reagentes (DMPD e $\left.\mathrm{Fe}(\mathrm{CN})_{6}{ }^{3-}\right)$ no percurso analítico à vazão de 3,4

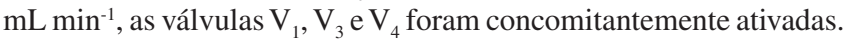
Ao aplicar essa estratégia, foi garantido que enquanto as alíquotas das soluções da amostra e dos reagentes eram introduzidas no percurso analítico, o fluxo do transportador estaria interrompido, uma vez que $V_{1}$ esteve sempre ativada enquanto $V_{2}, V_{3}$ e $V_{4}$ também estiveram.

Como destacado no diagrama de tempo da Figura 2, o processo 
para a determinação da concentração de PTU nas soluções dos medicamentos, empregando o sistema multicomutado de análises em fluxo, ocorreu em quatro etapas executadas sequencialmente e cujas operações são descritas a seguir:

Etapa 1. Ativar simultaneamente as válvulas solenoides $\mathrm{V}_{1}$ e $\mathrm{V}_{2}$ e mantê-las nesse estado por $\left(\Delta \mathrm{T}_{2}\right)$ 1,5 $\mathrm{s}$. Nessa condição, o fluxo de água (transportador) no percurso analítico é interrompido para a inserção, na posição $x$, de uma alíquota da solução da amostra (ou solução de referência de PTU).

Etapa 2. Desativar simultaneamente as válvulas $\mathrm{V}_{1} \mathrm{e} \mathrm{V}_{2}$ para que a água flua a partir de $\mathrm{V}_{1}$, transportando a zona da amostra da posição $x$ até a confluência $y(10 \mathrm{~cm})$. Manter essa condição por $\left(\Delta \mathrm{T}_{1}\right) 1,0 \mathrm{~s}$.

Etapa 3. Ativar simultaneamente as válvulas $\mathrm{V}_{1}, \mathrm{~V}_{3}$ e $\mathrm{V}_{4}$ para interromper o fluxo de água (transportador) e introduzir as alíquotas do reagente cromogênico (solução de DMPD) e do oxidante (solução de $\mathrm{K}_{3} \mathrm{Fe}(\mathrm{CN})_{6}$ ), a partir das válvulas solenoides $\mathrm{V}_{3}$ e $\mathrm{V}_{4}$, respectivamente. Para adicionar as alíquotas dos reagentes, manter $\mathrm{V}_{1}, \mathrm{~V}_{3} \mathrm{e}_{4}$ ativadas por $\left(\Delta \mathrm{T}_{3}=\Delta \mathrm{T}_{4}\right) 0,75 \mathrm{~s}$.

Etapa 4. Desativar concomitantemente as válvulas $\mathrm{V}_{1}, \mathrm{~V}_{3} \mathrm{e}$ $\mathrm{V}_{4}$. Manter todas as válvulas desativadas $\left(\Delta \mathrm{T}_{1}\right)$ por $60 \mathrm{~s}$, de modo a garantir o transporte da zona de reação da confluência passando pela bobina de mistura $\left(B_{1}\right)$ até atingir o detector $(475 \mathrm{~nm})$, bem como para o recondicionamento das linhas para uma nova tomada de alíquota de amostra.

Para a avaliação de possíveis erros sistemáticos resultantes da aplicação do procedimento de análises em fluxo proposto, foram determinadas em triplicata a concentração de PTU na matéria-prima aplicando o método espectrofotométrico de análises em fluxo proposto e o método volumétrico de referência descrito nas farmacopeias americana e europeia. ${ }^{22,23}$ Para a determinação de PTU em amostras do insumo pelo método de referência (titulação de neutralização com detecção potenciométrica do ponto final) foram tomadas $300 \mathrm{mg}$ da matéria-prima e, após a adição de $30 \mathrm{~mL}$ de solução de $\mathrm{NaOH}$ 0,1 mol $\mathrm{L}^{-1}$, a mistura foi levada à ebulição sob agitação para hidrólise do PTU. Em seguida, foram adicionados à mistura $50 \mathrm{~mL}$ de solução $0,1 \mathrm{~mol} \mathrm{~L}^{-1}$ de $\mathrm{AgNO}_{3}$ mantendo o aquecimento por $5 \mathrm{~min}$, visando a precipitação de compostos oriundos da degradação do PTU. Após resfriamento, o ácido sulfúrico gerado em solução após a hidrólise alcalina foi titulado contra solução padronizada $0,1000 \mathrm{~mol} \mathrm{~L}^{-1}$ de $\mathrm{NaOH}$.

\section{RESULTADOS E DISCUSSÃO}

\section{Efeito das condições reacionais sobre o equilíbrio tautomérico do propiltiouracil}

Para iniciar as investigações foi estabelecida uma sequência de assertivas: uma das formas tautoméricas do PTU é tiólica; os tióis podem reagir, como os sulfetos, com o radical iminoquinona produzido pela oxidação do DMPD; ${ }^{24}$ a reação entre sulfetos e DMPD leva à formação de corante fenotiazínico e, corantes apresentam elevada absortividade; induzindo a afirmar que o produto gerado pela reação entre a forma tiólica do PTU e a espécie radicalar produzida pela oxidação do DMPD será corado e apresentará alta absortividade.

Foram realizados estudos preliminares explorando a interação entre o DMPD e PTU em meio ácido ( $\mathrm{HCl}$ 0,2 $\left.\mathrm{mol} \mathrm{L}^{-1}\right)$ e empregando $\mathrm{Fe}^{3+}$ como oxidante, mimetizando as condições de reação do sulfeto com o radical iminoquinona em meio ácido. ${ }^{24}$ Contudo, o período necessário para a reação entre o DMPD e o PTU em meio $0,2 \mathrm{~mol} \mathrm{~L}^{-1}$ de $\mathrm{HCl}$ mostrou-se muita longo, o que compromete sua aplicação em rotinas de laboratórios de controle de qualidade. Em meio 0,2 mol L-1 de $\mathrm{HCl}$ foi necessário aguardar mais de 25 min para a produção de quantidade apreciável do produto. Em contrapartida, o período necessário para completar a reação em meio alcalino foi curto; o que é interessante, do ponto de vista das análises químicas. Porém, ao alterar as condições do meio reacional de ácido para alcalino foi necessário também substituir o agente oxidante. Assim, o $\mathrm{Fe}^{3+}$ foi substituído por solução de $\mathrm{Fe}(\mathrm{CN})_{6}{ }^{3-}$. Nos estudos preliminares executados em batelada e em meio alcalino foi constatado que o sinal gerado, logo após a mistura dos reagentes, era muito elevado (Figura 3). Adicionalmente, foi constatada (Figura 3) a diminuição do sinal analítico com o tempo após a mistura dos reagentes em meio alcalino e que essa diminuição do sinal analítico foi mais expressiva para reações em meios mais alcalinos. Essas constatações levaram a crer que a reação entre o radical iminoquinona e o PTU em meio alcalino produzia um intermediário instável, mas com elevada absortividade molar. Realizou-se um estudo mais detalhado sobre a influência do $\mathrm{pH}$ do meio reacional na estabilidade do produto da reação DMPD/Fe $(\mathrm{CN}){ }_{6}^{3-}$ com PTU e os dados são apresentados na Figura 3. O comportamento observado nessa etapa da investigação deve estar associado com a predominância entre os tautômeros do PTU para diferentes condições de alcalinidade do meio. Os resultados obtidos indicaram que em meios de maior acidez, as formas não tiólicas do PTU foram predominantes (Figura 1; estruturas I e II). Dessa forma, a interação entre o radical iminoquinona gerado $\left(\mathrm{DMPD}^{\bullet+}\right) \mathrm{e}$ o grupamento tiólico ocorreria em menor extensão, sendo necessário o deslocamento do equilíbrio tautomêrico para garantir a formação de quantidade apreciável do produto a ser espectrofotometricamente monitorado. Por outro lado, em meio mais fortemente alcalino, o equilíbrio tautomérico seria deslocado no sentido da formação da espécie tiólica e de sua base conjugada (Figura 1; estruturas III e IV), o que conduziria a uma diminuição do período necessário para a formação do produto da reação com o radical iminoquinona.

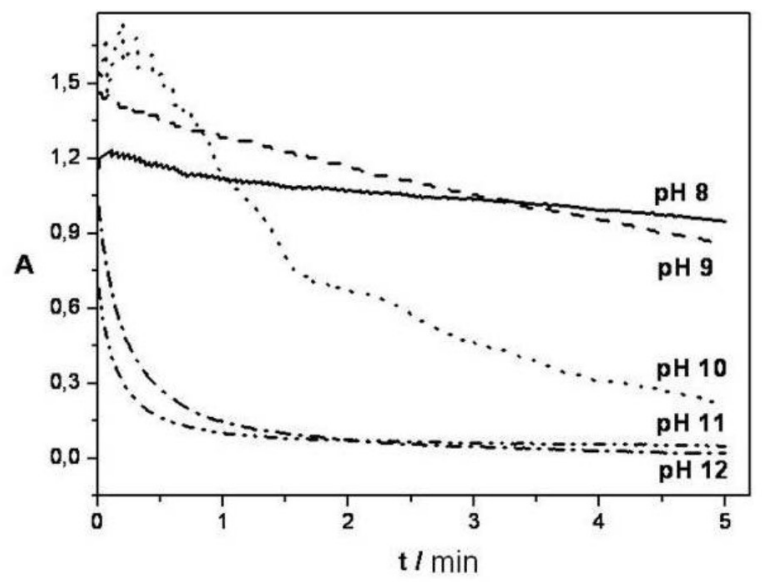

Figura 3. Perfis de decaimento do sinal analítico para reação entre solução de PTU (20 mg $\left.\mathrm{L}^{-1}\right)$, solução de DMPD $\left(1,5 \times 10^{-3} \mathrm{~mol} \mathrm{~L}^{-1}\right)$ e solução 0,5\% $(\mathrm{m} / \mathrm{v})$ de $\mathrm{Fe}(\mathrm{CN})_{6}^{3-}$ para diferentes valores de $\mathrm{pH}$

A instabilidade da espécie sob monitoração produzida pela reação do PTU em meio alcalino levou à necessidade de se empregar um sistema automatizado de análise. Neste sentido, foi necessário diminuir o tempo de permanência da zona de amostra após a adição das alíquotas dos reagentes para garantir a formação da espécie de interesse e minimizar a sua degradação (Figura 3). O emprego de sistema multicomutado de análises em fluxo propiciou não somente o aumento na taxa de amostragem, como também aumento da sensibilidade. Assim, para as avaliações posteriores, o $\mathrm{pH}$ do meio reacional foi fixado em 10 .

\section{Avaliação das condições experimentais}

Os primeiros parâmetros avaliados foram as concentrações das soluções de DMPD e de $\mathrm{Fe}(\mathrm{CN})_{6}{ }_{6}^{3-}$, mantendo invariável a concentração 
da solução de referência em $20 \mathrm{mg} \mathrm{L}^{-1}$ de PTU. O efeito da concentração de DMPD sobre o sinal analítico foi avaliado na faixa de 0,1 a 1,5 mmol L ${ }^{-1}$; enquanto que, para a solução do oxidante, a faixa de concentração variou de 0,05 a $0,5 \%(\mathrm{~m} / \mathrm{v})$. No sistema multicomutado de análises em fluxo não foi observado aumento significativo do sinal analítico para $95 \%$ de confiança, para concentrações superiores a 0,5 mmol L-1 de DMPD. Logo, a concentração de 0,5 mmol DMPD L ${ }^{-1}$ foi selecionada para estudos seguintes. A concentração do oxidante que levou aos melhores resultados foi $0,15 \%(\mathrm{~m} / \mathrm{v})$. Para concentrações da solução de $\mathrm{Fe}(\mathrm{CN})_{6}{ }^{3-}$ maiores que $0,15 \%(\mathrm{~m} / \mathrm{v})$ foi observada diminuição do sinal líquido. Isso ocorreu devido ao aumento considerável da concentração da forma oxidada do DMPD no branco. A forma oxidada do reagente cromogênico apresenta máximo de absorção em região próxima ao do máximo de absorção do produto sob monitoração (475 nm).

O efeito do volume das alíquotas dos reagentes sobre o sinal analítico foi também avaliado. A concepção do sistema multicomutado de análises em fluxo previa a inserção simultânea das alíquotas dos reagentes (DMPD e $\mathrm{Fe}(\mathrm{CN})_{6}{ }^{3-}$ ). Portanto, o intervalo de tempo de acionamento das válvulas responsáveis pela introdução das alíquotas de DMPD e Fe $(\mathrm{CN})_{6}{ }_{6}^{3-}$ foi igual. Foi avaliado o volume dos reagentes (solução de DMPD e solução de $\mathrm{Fe}(\mathrm{CN})_{6}{ }^{3}{ }^{-}$) entre 21,25 e 56,5 $\mu \mathrm{L}$. Observou-se que para longos intervalos de tempo de acionamento das válvulas dos reagentes houve a diminuição do sinal líquido. Logo, para os ensaios posteriores, foram fixados em $42,5 \mu \mathrm{L}$ os volumes das soluções de DMPD e de $\mathrm{Fe}(\mathrm{CN})_{6}{ }^{3-}$. Nas condições estabelecidas, o sistema multicomutado proposto de análises em fluxo para a determinação de PTU produziu 1,2 mL de volume residual por determinação.

$\mathrm{Na}$ avaliação do desempenho analítico considerou-se o comprimento da bobina de reação $\left(\mathrm{B}_{1}\right)$ como um parâmetro crítico. Essa importância foi justificada pelo fato do método de determinação da concentração de PTU ser baseado na monitoração de uma espécie intermediária, instável e que, portanto, se degrada enquanto é endereçada para o detector. Em outras palavras, pôde ser antecipado que quanto maior o tempo de permanência do produto da reação no percurso analítico, menor seria a sensibilidade do método. $\mathrm{O}$ efeito do comprimento da bobina de reação (de 40 a $140 \mathrm{~cm}$ ) sobre o sinal analítico foi estudado e é mostrado na Figura 4. Foi observado aumento significativo no sinal analítico (ca. 90\%) quando o comprimento de $B_{1}$ variou de 40 para $60 \mathrm{~cm}$, evidenciando, nos primeiros instantes após a mistura, a prevalência do processo de deslocamento do equilíbrio tautomérico do PTU no sentido de aumentar a proporção de sua forma tiólica. Também pode ser observada (Figura 4) diminuição do sinal analítico em $70 \%$ com o aumento do comprimento da bobina de reação de 60 para $100 \mathrm{~cm}$, o que indica que quando a zona de amostra atingiu o detector o fenômeno de degradação do produto da reação no percurso analítico foi o prevalente. Logo, para os ensaios posteriores foi empregada bobina de reação com $60 \mathrm{~cm}$.

Finalmente, avaliou-se o efeito da vazão do fluido transportador sobre o sinal analítico. A investigação foi feita para vazões entre 2,0 e 4,6 mL min ${ }^{-1}$, sendo observado aumento do sinal analítico com o aumento da vazão de 2,0 para $2,8 \mathrm{~mL} \mathrm{~min}^{-1}$. Similarmente ao que havia sido constatado na avaliação do efeito do comprimento da bobina de reação sobre o sinal analítico, observou-se diminuição linear do sinal analítico com o aumento da vazão do transportador a partir de 2,8 $\mathrm{mL} \mathrm{min}^{-1}$. Assim, para o procedimento proposto foi fixada a vazão de $2,8 \mathrm{~mL} \mathrm{~min}^{-1}$ para o fluido transportador, garantindo dessa forma frequência analítica superior a 55 determinações por hora.

As avaliações dos efeitos da variação da vazão do transportador e do tamanho da bobina de reação sobre o sinal analítico levaram a resultados concordantes e ratificaram a influência do tempo de residência sobre o desempenho analítico. Os resultados demonstraram que o maior sinal analítico líquido foi obtido para vazão do

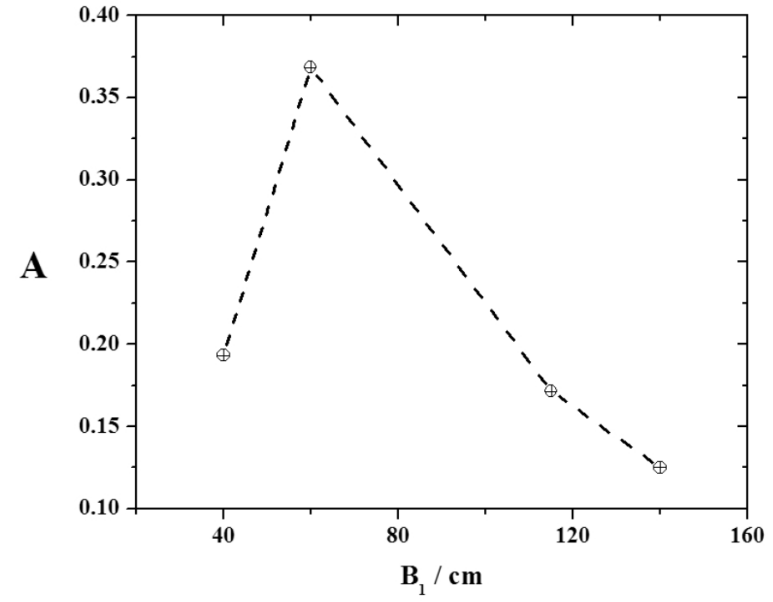

Figura 4. Avaliação do efeito do comprimento do percurso analítico sobre o sinal para o sistema multicomutado de análises em fluxo $(n=3)$. Condições: 42,5 $\mu \mathrm{L}$ de $\mathrm{Fe}(\mathrm{CN})_{6}{ }^{3-} 0,15 \%(\mathrm{~m} / \mathrm{v}), 42,5 \mu \mathrm{L} \mathrm{DMPD} 0,5 \mathrm{mmol} \mathrm{L}^{-1}$ e $170 \mu \mathrm{L}$ de PTU $20 \mathrm{mg} \mathrm{L}^{-1}$, vazão transportador $=2,8 \mathrm{~mL} \mathrm{~min}^{-1}$

transportador igual a $2,8 \mathrm{~mL} \mathrm{~min}^{-1}$, indicando que dois processos antagônicos (produção e degradação) são os responsáveis pelo desempenho desse método de análíse. Em outras palavras, para adequar o desempenho do procedimento analítico foi imperativo assegurar: intervalo de tempo suficientemente longo para que houvesse a máxima conversão dos tautômeros do PTU para sua forma tiólica pelo deslocamento do equilíbrio tautomérico; e, uma vez formado o produto da reação que o intervalo de tempo fosse suficientemente curto para a medida da espécie intermediária, minimizando sua degradação em linha. Assim, os processos foram separadamente avaliados para definir os valores dos parâmetros do sistema (comprimento da bobina de reação) e de fluxo (vazão do carregador) que garantissem o maior sinal líquido. Nesse contexto, o controle do tempo para cada operação assumiu um caráter preponderante, uma vez que variações no tempo de permanência da zona de amostra no percurso analítico também prejudicariam a precisão. Assim, foi garantida, por sincronização, a constância da vazão e dos intervalos de tempo para a amostragem das alíquotas das soluções (amostra e reagentes) e para o transporte da zona de amostra até o detector. Um controle menos rígido dos períodos de ativação e desativação das válvulas solenoides $\left(\mathrm{V}_{1}, \mathrm{~V}_{2}\right.$, $\mathrm{V}_{3}$ e $\mathrm{V}_{4}$ ) comprometeria significativamente a precisão do método; enquanto que a escolha inadequada dos intervalos de tempo para as tomadas de alíquota de amostra $\left(\Delta \mathrm{T}_{2}\right)$ e reagentes $\left(\Delta \mathrm{T}_{3}, \Delta \mathrm{T}_{4}\right)$, bem como do tempo de residência $\left(\Delta \mathrm{T}_{1}\right)$, afetaria à sensibilidade analítica e, consequentemente, os limites de detecção e de quantificação.

\section{Validação e aplicação do procedimento analítico}

A acurácia do método proposto foi avaliada pela comparação dos resultados de concentração de PTU na matéria-prima obtidos empregando o procedimento desenvolvido neste trabalho e o método de referência, o qual não é recomendado para a determinação de PTU em medicamentos. Neste sentido, os métodos proposto e de referência foram aplicados em quintuplicata à matéria-prima, sendo obtidos os valores de $292 \pm 4$ e $301 \pm 7 \mathrm{mg}$, respectivamente. Os resultados obtidos para a matéria-prima pelos dois métodos foram concordantes, aplicando-se teste $t$ para 95\% de confiança. Entretanto, deve ser ressaltado que não foi possível a aplicação do método volumétrico para a quantificação de PTU em amostras de medicamentos, devido à presença de interferentes, como o amido. Portanto, para avaliar possíveis efeitos de matriz sobre o procedimento analítico proposto foram realizados ensaios de recuperação. Os ensaios de recuperação 
foram executados com as amostras de medicamento, pela adição de quantidades distintas e conhecidas do padrão. Pelos resultados obtidos, a recuperação variou entre 92 e 104\%, evidenciando a ausência de erros sistemáticos no procedimento multicomutado de análises em fluxo proposto.

Na Tabela 1 são apresentados outros parâmetros de desempenho relacionados ao método analítico proposto. Os limites de detecção $(3 \sigma)$ e de quantificação $(10 \sigma)$ para o procedimento analítico proposto foram estimados para 10 determinações de PTU em brancos independentes em 0,11 e $0,37 \mathrm{mg} \mathrm{L}^{-1}$, respectivamente. A linearidade do método analítico foi avaliada $\left(S_{a}=0,013 \cdot C_{P T U}+0,002\right)$ e ficou evidenciada a resposta linear $(\mathrm{R}=0,9996)$ para concentrações do analito $\left(C_{P T U}\right)$ de 2,5 a $20 \mathrm{mg} \mathrm{L}^{-1}$. A precisão do método foi avaliada em $1,4 \%(\mathrm{n}=5)$ para concentração de PTU de $5 \mathrm{mg} \mathrm{L}^{-1}$. O método desenvolvido apresentou sensibilidade igual a $0,013 \mathrm{~L} \mathrm{mg}^{-1}$.

Uma impureza comumente encontrada em medicamentos à base de PTU é a tioureia. ${ }^{23}$ Assim, foi avaliado o efeito desse composto como possível interferente para o método de determinação de PTU. Os ensaios foram realizados em soluções $10 \mathrm{mg} \mathrm{L}^{-1}$ de PTU contendo tioureia na faixa de concentração entre 0 e $150 \mathrm{mg} \mathrm{L}^{-1}$. Não foram observadas variações $>5 \%$ do sinal analítico, mesmo para solução com concentração de tioureia $150 \mathrm{mg} \mathrm{L}^{-1}$.

Excipientes comumente presentes nas formulações farmacêuticas de PTU, como glicolato de amido sódico, povidona, estearato de magnésio, amido e carbonato de cálcio não apresentaram interferfência
Tabela 1. Características do desempenho do procedimento multicomutado de análises em fluxo para determinação espectrofotométrica de propiltiouracil após dissolução em água

\begin{tabular}{lc}
\hline Figura de mérito & Método proposto \\
\hline Concentração máxima do analito, $\mathrm{mg} \mathrm{L}^{-1}$ & 20 \\
Sensibilidade, $\mathrm{L} \mathrm{mg}^{-1}$ & 0,013 \\
Limite de detecção, $\mathrm{mg} \mathrm{L}^{-1}$ & 0,11 \\
Limite de quantificação, $\mathrm{mg} \mathrm{L}^{-1}$ & 0,37 \\
Desvio padrão relativo $\left(20 \mathrm{mg} \mathrm{L}^{-1}, \mathrm{n}=5\right)$ & $1,4 \%$ \\
Taxa de amostragem, $\mathrm{h}^{-1}$ & 55 \\
\hline
\end{tabular}

na metodologia desenvolvida em função da baixa solubilidade dessas espécies em água e metanol.

Parâmetros de desempenho do método proposto foram comparados com os reportados para outros processos de determinação de PTU anteriormente publicados ${ }^{2-7}$ e essa comparação é apresentada na Tabela 2

O procedimento desenvolvido foi aplicado a três formulações farmacêuticas produzidas por diferentes laboratórios farmacêuticos brasileiros. Os resultados obtidos são apresentados na Tabela 3, sendo possível observar que todas as amostras estavam em conformidade com o estabelecido pela legislação da ANVISA. ${ }^{25}$

Tabela 2. Parâmetros de desempenho de diferentes métodos de determinação de PTU em variadas matrizes

\begin{tabular}{|c|c|c|c|c|c|}
\hline Princípios dos métodos & Matrizes & Faixa linear $\left(\mathrm{mg} \mathrm{L}^{-1}\right)$ & $\mathrm{LD}\left(\mathrm{mg} \mathrm{L}^{-1}\right)$ & Recuperação (\%) & Ref. \\
\hline$\overline{\mathrm{EAM}}$ & Medicamentos & $1,3-14$ & 0,41 & n.i & 2 \\
\hline Cinético / EAM & Medicamento e insumo & $0,006-0,033$ & 0,004 & n.i & 3 \\
\hline EAM & Medicamento e insumo & $0,39-4,2$ & n.i & n.i & 4 \\
\hline Extração por solvente / EAM & Fluído biológico & $0,5-10$ & n.i & $92-109$ & 5 \\
\hline CLAE / EAM & Fluído biológico & $0,31-40$ & 0,05 & $94-103$ & 6 \\
\hline Cinético / Voltamétrico & Sintética & $0,02-1,7$ & 0,008 & n.i & 7 \\
\hline EAM & Medicamento e insumo & $0,37-20$ & 0,11 & 91-104 & Este trabalho \\
\hline
\end{tabular}

$\mathrm{LD}=$ limite de detecção $(3 \sigma)$. EAM = espectrofotometria. CLAE = cromatografia líquida de alta eficiência. $n . i$ = não informado

Tabela 3. Determinação de PTU em amostras de medicamentos empregando sistema multicomutado de análises em fluxo proposto. Valor declarado igual a $100 \mathrm{mg}$ de PTU por comprimido. Resultados expressos em termos de médias e dos respectivos desvios padrão da média $(\mathrm{n}=3)$

\begin{tabular}{cc}
\hline Amostra & Método proposto $(\mathrm{mg})$ \\
\hline A & $93 \pm 5$ \\
B & $93 \pm 5$ \\
C & $100 \pm 2$ \\
\hline
\end{tabular}

\section{CONCLUSÕES}

Os resultados apresentados evidenciaram que, a despeito das diferentes formas canônicas do PTU e da alta instabilidade da espécie sob monitoração, o procedimento em fluxo desenvolvido possibilitou a determinação espectrofotométrica desse fármaco em soluções aquosas. Apesar da elevada instabilidade do intermediário formado na reação entre os radicais iminoquinonas e a forma tiólica do fármaco, o método demonstrou ser muito seletivo e sensível para as determinações nas amostras investigadas (insumos e medicamentos). A implementação do método analítico em um sistema automatizado de análises, como o sistema multicomutado aqui projetado, foi justificada para garantir a precisão nos resultados.

\section{AGRADECIMENTOS}

À CAPES, CNPq e FAPESB pelo apoio.

\section{REFERÊNCIAS}

1. Sacré, P.-Y.; Deconinck, E.; Daszykowski, M.; Courselle, P.; Vancauwenberghe, R.; Chiap, P.; Crommen, J.; De Beer, J. O.; Anal. Chim. Acta 2011, 701, 224.

2. Stankovic, B.; Jovanovic, T.; Masic, S.; Koricanac, Z.; Farmaco 1996, 51,679 .

3. Barzegar, M.; Rahmani, A.; Jabbari, A.; Mousavi, M. F.; Pharmazie 2003, 58, 114 .

4. Skowron, M.; Ciesielski, W.; Chem. Anal. 2009, 54, 743.

5. Ratliff, C. R.; Gilliland, P. F.; Hall, F. F.; Clin. Chem. 1972, 18, 1373.

6. Spadaro, A. C. C.; Duarte, C. G.; Polizello, A. C. M.; Assis-Pandochi, A. I.; J. Pharm. Biomed. Anal. 2000, 23, 237.

7. Shahrokhian, S.; Saberi, R. S.; Int. J. Electrochem. Sci. 2007, 2, 158

8. Hegedüs, L.; Endocrinol. Metab. Clin. North Am. 2009, 38, 355.

9. Goldstein, G. L.; Daun, H. B.; Tepper, J.; Physiol. Behav. 2007, 90, 809.

10. Morrison, R. T.; Boyd, R. N.; Química Orgânica, $9^{\mathrm{a}}$ ed., Fundação Calouste Gulbenkian: Lisboa, 1990.

11. Raczyฑ́ska, E. D.; Zientara, K.; Kolczyńska, K.; Stępniewsk, T.; J. Mol. Struct.: Theochem. 2009, 894, 103. 
12. Spisso, B. F.; Araújo Júnior, M. A. G.; Monteiro, M. A.; Lima, A. M. B.; Luiz, R. A.; Pereira, M. U. ; Nóbrega, A. W.; Anal. Chim. Acta 2009, 656,72 .

13. Cherlet, M.; Croubels, S.; De Backer, P.; J. Chromatogr., A 2006, 1102, 116.

14. Huang, L.; Huang, Y.; Chi, Y.; Lin, J.; Yu, L.; Xu, L.; Chen, G.; J. Chromatogr., A 2007, 1175, 283.

15. Wojnarowska, Z.; Wlodarczyk, P.; Kaminski, K.; Grzybowska, K.; Hawelek, L.; Paluch, M.; J. Chem. Phys. 2010, 133, 094507-1.

16. Størmer, F. C.; Janak, K.; Mycologist 2004, 18, 114.

17. Fortes, P. R.; Feres, M. A.; Zagatto, E. A. G.; Lima, J. L. F. C.; Talanta 2010, 81, 1409.

18. Nalewajko-Sieliwoniuk, E.; Tarasewicz, I.; Kojło, A.; Anal. Chim. Acta 2010, 668, 19.
19. Pinyoua, P.; Youngvisesb, N.; Jakmunee, J.; Talanta 2011, 84, 745.

20. Ruzicka, J.; Hansen, E. H.; Anal. Chim. Acta 1975, 78, 145.

21. Rocha, F. R. P.; Reis, B. F.; Zagatto, E. A. G.; Lima, J. L. F. C.; Lapa, R. A. S.; Santos, J. L. M.; Anal. Chim. Acta 2002, 466, 125.

22. United States Pharmacopeia; United States Pharmacopeia Convention, $25^{\text {th }}$ ed., 2002.

23. European Pharmacopeia; Pharmaeuropa Quartely Forum Publication, $4^{\text {th }}$ ed., 2001.

24. Chagas, M. P.; Santos, J. C. C.; Santos, E. B. G. N.; Oliveira, T. D.; Korn, M.; J. Braz. Chem. Soc. 2009, 20, 1646.

25. http://www.anvisa.gov.br/medicamentos/legis/01_05_re_comentada.pdf, acessada em Novembro 2012. 